where

$$
\alpha=\sqrt{b^{2}-c^{2}}, \quad \beta=\sqrt{c^{2}-a^{2}}, \quad \gamma=\sqrt{a^{2}-b^{2}}
$$

Transform to a new system of coördinates $x_{1}, x_{2}, x_{3}, x_{4}$, by means of the equations

$$
\begin{aligned}
& x_{1}=(c-b)\left[a \alpha \beta x-\left(b \beta^{2}-c \gamma^{2}\right) y+i a c \alpha \gamma w\right], \\
& x_{2}=(b+c)\left[a \alpha \beta x-\left(b \beta^{2}+c \gamma^{2}\right) y+i a c \alpha \gamma w\right], \\
& x_{3}=(a+c)[a \alpha x-b \beta y+c \gamma z], \\
& x_{4}=(c-a)[a \alpha x-b \beta y-c \gamma z] .
\end{aligned}
$$

With respect to this new system, the coördinates of the six chosen nodes are $(1,0,0,0),(0,1,0,0),(0,0,1,0)$, $(0,0,0,1),\left(e_{1}, e_{2}, e_{3}, e_{4}\right),\left(e_{2}, e_{1}, e_{4}, e_{3}\right)$, where

$$
\begin{array}{ll}
e_{1}=\beta\left(b^{2}-c^{2}\right)(c-a), & e_{3}=\left(a^{2}-c^{2}\right)(c+b), \\
e_{2}=\beta\left(b^{2}-c^{2}\right)(c+a), & e_{4}=\left(a^{2}-c^{2}\right)(c-b) .
\end{array}
$$

These six points form an involution of the kind described. (See Annals of Mathematics, vol. 11, p. 159.)

\title{
NOTE ON INTEGRATING FACTORS.
}

BY MR. PAUL SAUREL.

( Read before the American Mathematical Society at the Meeting of February $26,1898$. )

IF the differential equation

$$
X_{1} d x_{1}+X_{2} d x_{2}+\cdots+X_{n} d x_{n}=0, \quad n \equiv 3,
$$

be integrable, and if $u=$ constant be the integral of this equation, then, as is well known, there exists a function $\boldsymbol{M}$ such that

And as

$$
d u \equiv M X_{1} d x_{1}+M X_{2} d x_{2}+\cdots+M X_{n} d x_{n}
$$

$$
\frac{\partial u}{\partial x_{1}} \equiv M X_{1}, \quad \frac{\partial u}{\partial x_{2}} \equiv M X_{2}, \cdots \frac{\partial u}{\partial x_{n}} \equiv M X_{n},
$$


and

$$
\frac{\partial^{2} u}{\partial x_{r} \partial x_{s}} \equiv \frac{\partial^{2} u}{\partial x_{s} \partial x_{r}}
$$

$M$ must satisfy the conditions

$$
\frac{\partial}{\partial x_{r}}\left(M X_{s}\right) \equiv \frac{\partial}{\partial x_{s}}\left(M X_{r}\right)
$$

The object of this note is to establish the conditions for the existence of an integrating factor which shall contain but one of the variables, and to show that such an integrating factor, if it exists, is unique.

If $M$ be a function of only one of the variables, $x_{1}$ for example, the conditions ( 1 ) yield the $n-1$ equations

$$
M \frac{\partial X_{1}}{\partial x_{r}}=M \frac{\partial X_{r}}{\partial x_{1}}+X_{r} \frac{d M}{d x_{1}}, \quad r=2,3, \cdots, n ;
$$

or, in better form,

$$
\frac{\frac{\partial X_{1}}{\partial x_{r}}-\frac{\partial X_{r}}{\partial x_{1}}}{X_{r}}=\frac{\frac{d M}{d x_{1}}}{M}, \quad r=2,3, \cdots, n ;
$$

and the $\frac{1}{2}(n-1)(n-2)$ equations

or

$$
M \frac{\partial X_{s}}{\partial x_{r}}=M \frac{\partial X_{r}}{\partial x_{s}}
$$

$$
\frac{\partial X_{s}}{\partial x_{r}}=\frac{\partial X_{r}}{\partial x_{s}}, \quad r \neq s ; \quad r, s=2,3, \cdots, n .
$$

In equations ( $I$ ) the right-hand member is a function of $x_{1}$ alone, and the left-hand members are therefore all equal to the same function of $x_{1}$.

Thus the conditions necessary for the existence of an integrating factor which shall be a function of $x_{1}$ alone, are that the left-hand members of equations (I) shall be identically equal to one and the same function of $x_{1}$, and that equations (II) shall hold identically, $-\frac{1}{2} n(n-1)$ conditions in all.

These are also the sufficient conditions. For, if they be

satisfied, put

$$
\frac{d M}{d x_{1}}
$$


equal to one of the equal expressions

$$
\frac{\frac{\partial X_{1}}{\partial x_{r}}-\frac{\partial X_{r}}{\partial x_{1}}}{X_{r}}, \quad r=2,3, \cdots, n,
$$

and integrate ; then is $M$, except as to an arbitrary constant factor, uniquely determined. And the function thus determined is an integrating factor, for the conditions (1) are all satisfied in virtue of equations (I) and (II).

If the proof given above be applied to the case of an equation containing two variables, it will show that the necessary and sufficient condition for an integrating factor containing $x_{1}$ alone is that

$$
\frac{\frac{\partial X_{1}}{\partial x_{2}}-\frac{\partial X_{2}}{\partial x_{1}}}{X_{2}}=f_{1}\left(x_{1}\right) \text {. }
$$

If at the same time the condition for an integrating factor containing $x_{2}$ alone be satisfied, viz :

$$
\frac{\frac{\partial X_{2}}{\partial x_{1}}-\frac{\partial X_{1}}{\partial x_{2}}}{X_{1}}=f_{2}\left(x_{2}\right),
$$

then

$$
\frac{X_{1}}{f_{1}\left(x_{1}\right)}=-\frac{X_{2}}{f_{2}\left(x_{2}\right)},
$$

and the differential equation may be put into the form

$$
f_{1}\left(x_{1}\right) d x_{1}-f_{2}\left(x_{2}\right) d x_{2}=0
$$

in which the variables are separate.

The uniqueness of the integrating factor containing but one of the variables may also be obtained very simply from the known theorem that the ratio of any two integrating factors is an integral of the differential equation. For, since this ratio contains all the variables, if only one of the variables appears in the first integrating factor, the second must contain all the remaining variables, and may contain all the variables.

\section{New YoRK,}

January, 1898. 